\title{
Anticoagulation in COVID - 19: An Update
}

\author{
Nishant R Tiwari ${ }^{1 *}$, Khalid I Khatib², Subhal B Dixit ${ }^{3}$, Prajay K Rathore ${ }^{4}$, Sameer Melinkeri ${ }^{5}$, \\ Abhijeet Ganapule ${ }^{6}$, Kapil S Borawake, Ujwala Mhatre ${ }^{8}$ \\ ${ }^{1}$ Byramjee Jeejeebhoy Medical College and Sassoon General Hospital, Pune, Maharashtra, India \\ 2 Smt Kashibai Navale Medical College and General Hospital, Pune, India \\ 3 Sanjeevan Hospital, Pune, India \\ ${ }^{4}$ Danbury Hospital, Danbury, USA \\ 5 Deenanath Mangeshkar Hospital and Research Centre, Pune, India \\ ${ }^{6}$ Niche Hematology Care, Kolhapur, India \\ 7 Prayag Hospital, Pune, India \\ 8 Nanavati Hospital, Mumbai, India
}

\begin{abstract}
The novel coronavirus disease, 2019 (COVID - 19) evolved as an unprecedented pandemic. The severe acute respiratory syndrome-corona virus-2 (SARS-CoV-2) infection has been associated with significantly deranged coagulation parameters and increased incidence of thrombotic events. Deranged coagulation parameters, such as D-dimers and fibrin degradation products, can indicate a poor prognosis, and their measurement will help stratify the patients according to the disease severity, need of intensive care unit admission, and prediction of the clinical course. Gaps in understanding the natural history of the disease cause difficulties in tailoring therapies and optimizing the management of patients. Lack of specific treatment further complicates this situation. While thrombotic events can cause significant morbidity and mortality in patients, a focused approach to the prevention and treatment of venous thromboembolism (VTE) can, to a great extent, decrease the disease burden caused by thrombotic diseases. Pharmacological prophylactic anticoagulants and mechanical therapies such as pneumatic compression devices can help prevent venous thromboembolism and other thrombotic events. Thrombotic events due to COVID-19, their prevention and management, are the focus of this paper, with the prospect of providing insights into this relatively unexplored area.
\end{abstract}

Keywords: COVID-19, anticoagulation, venous thromboembolism, SARS-CoV-2, intensive care unit

Received: 14 July 2020 / Accepted: 16 September 2020

\section{INTRODUCTION}

SARS-CoV-2 has glycoprotein spikes on its capsid, designated as $S$ protein, which helps it engage specific receptors on the cells of target organisms. For human infections, this receptor happens to be the angiotensinconverting enzyme 2 (ACE) receptor of the respiratory epithelium [1]. This receptor is expressed by many other cells of the body, including vascular endothelium and cardiac myocytes.

The virus replicates after it enters into these cells and causes severe acute inflammation and cellular lysis. This inflammatory state initiates a cytokine storm with the release of many pro-inflammatory cytokines, for example, interleukin-6 (IL-6). As a consequence of this, patients infected with COVID-19 are at high risk of developing acute respiratory distress syndrome (ARDS), multiple organ failure, and shock [2].
Thrombotic events in COVID-19 patients are reported in the literature due to its potential to cause endotheliopathy [3].

In this review, the abnormal laboratory parameters, the strategies for prevention and treatment of thrombotic events, and associated anticoagulant therapy in patients with COVID-19 associated illness, are considered.

\section{INTENSIVE CARE TREATMENT AND COVID - 19}

The literature from Italy and China indicated high mortality amongst COVID-19 patients admitted to intensive care units (ICU). Further, its rampant spread led to a rapid rise in numbers of critically ill patients with COVID-19. It caused the overburdening of ICUs and entire health care systems in general $[4,5]$. 
A case series from the USA reported a fatality rate of $50 \%$ in COVID-19 patients admitted to ICUs. Patients with comorbidities such as diabetes mellitus (DM), asthma, and chronic kidney disease (CKD) were more prone to the development of serious illness. About $71 \%$ of patients in this study required vasopressors for the maintenance of blood pressure, while none had coinfections with bacteria or other viruses. It led to a hypothesis that the circulatory shock may be directly related to COVID-19 [6].

This underlines the importance of optimized management of patients with COVID-19 related illnesses in ICUs.

The haemostatic pathway is an established component of innate immunity. The process of haemostasis is protective in infective states as it helps prevent the spread of the organisms and concentrates antimicrobial cells in the area of inflammation [7].

However, in states of severe infection like sepsis, when this process progresses out of control, widespread activation of coagulation pathways may occur, and a state of consumptive coagulopathy, manifesting as disseminated intravascular coagulation (DIC) can develop.

There are many theories as to the underlying mechanisms, with notable ones being bacterial polyphosphates activating platelets, endothelial expression of tissue factor because of exposure to cytokines, and the formation of neutrophil extracellular traps (NETs) [8].

Infection by SARS-CoV-2 is an inflammatory state evidenced by increased levels of C-reactive protein, IL-6, and an elevated erythrocyte sedimentation rate (ESR). The endothelial cells infected with viruses undergo lysis and attract antigen-presenting cells (APCs). These antigen-presenting cells then activate T-lymphocytes resulting in a propagative state of inflammation resulting in a widespread activation of platelets as well as the coagulation pathway, with the possibility of coagulation exceeding the capacity of clearance by natural anticoagulants [3].

\section{-THROMBOTIC RISK ASSOCIATED WITH COVID - 19}

Common and critical haematological abnormalities seen in COVID-19 patients were lymphocytopenia, thrombocytopenia, leukopenia, and elevated D-dimers [9]. Klok et al. (2020) evaluated the incidence of thrombotic complications in COVID-19 patients admitted to the ICU. They found the frequency of thrombotic complications in these patients to be $31 \%$ [10]. Complications were attributable to hypoxia, DIC, extreme inflammation, and immobilization due to the disease. Surprisingly, in this study, the patients who developed thrombotic events were already getting standard doses of prophylactic anticoagulation [10].

An autopsy series of confirmed COVID-19 cases demonstrated diffuse alveolar haemorrhage, microvascular thromboses, and marked extracellular fibrin deposition in the lungs [11]. A chinese study reported the incidence of DIC as $71.4 \%$ in patients who succumbed to COVID-19 pneumonia [12]. Klok et al. (2020) reevaluated the results of their previous study later in the year [10], and found, on longer follow-up, an even higher cumulative incidence of the composite outcome of thrombotic events at $49 \%$ [13].

A multicentre retrospective cohort study, studying thrombotic and bleeding manifestations of COVID-19 patients, recorded an overall thrombotic complication rate of $18.1 \%$ in critically ill patients. All but one patient in this study, who developed a thrombotic event, were getting a standard dose of prophylactic anticoagulation. The same study also recorded a major-bleeding event rate of $5.6 \%$ in critically ill patients [14]. In another retrospective cohort study on COVID-19 patients admitted to an ICU, the incidence of venous thromboembolism (VTE) was 25\% [15]. Moreover, patients who developed VTE were older, had lymphocytopenia, increased D-dimer, and a prolonged activated partial thromboplastin time (aPTT). This study also linked Tcell immune dysfunction to the increased incidence of VTE [15].

In a retrospective cohort study including critically ill COVID-19 patients, the incidence of VTE was 26.1\%; the risk was higher at $73 \%$ in patients who required extracorporeal membrane oxygenation (ECMO) support [16]. Also, the patients who had an episode of VTE were more likely to need mechanical ventilation and vasopressors. It implies that increased rates of VTE occur in patients having more severe disease [16].

In a prospective cohort study on the patients admitted to an ICU due to COVID-19 related illness, sixtyfour clinically significant thrombotic events occurred in 150 patients, with pulmonary embolism being the most commonly encountered thrombotic event [17]. In the same study, investigators also compared risks of thrombotic complications in COVID-19 related ARDS patients versus non-COVID-19 related ARDS patients. 
They found the risk of thrombotic events and pulmonary embolism was significantly higher in COVID-19 related ARDS patients, with an odds ratio of 2.6 (95\% $\mathrm{CI}=1.1$ to 6.1$)$ and $6.2(95 \% \mathrm{CI}=1.6$ to 23.4$)$ respectively [17].

A retrospective cohort study on twenty-six critically ill, mechanically ventilated, COVID-19 patients receiving eight prophylactic-doses plus eighteen therapeuticdose of anticoagulants reported an overall rate of VTE as $69 \%$. In this study, VTE occurred in all patients receiving prophylactic doses of anticoagulants, and in $56 \%$ of patients receiving therapeutic doses of anticoagulants [18].

A French prospective cohort study on critically ill, mechanically ventilated COVID-19 patients, screened patients for deep vein thrombosis (DVT) in the lower extremity and yielded similar results. The incidence of DVT, forty-eight -hours after admission to the ICU, was 79\% [19]. The importance of screening COVID-19 patients for DVT and their prompt management in the form of therapeutic-dose anticoagulation to prevent further morbidity and mortality was thus highlighted.

\section{- HAEMATOLOGICAL PARAMETERS IN COV- ID-19}

Derangements in haematological parameters like elevated D-dimers and fibrin degradation products (FDPs), thrombocytopenia, prolonged prothrombin time (PT), and aPTT are relatively common in COVID-19 patients [3].

Table 1 summarizes some of the haematological abnormalities seen in COVID-19 patient studies. From the interpretation of this data, the following can be deduced.

1. It is common to see deranged coagulation parameters, especially D-dimer and FDPs, in COVID-19 patients.

2. These derangements are, by and large, associated with a poorer prognosis and increased likelihood of ICU admission.

3. These parameters, if checked on admission to the hospital, can serve as a guide to triage patients, based on severity and risk of deterioration. Their serial monitoring may also help predict the clinical course of the disease and tailor the required therapy.

In a retrospective cohort study, looking at the causes of elevated D-dimers, investigators found that infec- tions were the most common cause of elevated D-dimers. Of the patients with infections having elevated $\mathrm{D}$-dimers, pneumonia was the cause in almost $2 / 3 \mathrm{rd}$ of cases [20]. This highlights the propensity of lung infections as the cause of elevated D-dimers in general. However, this also raises an important question - is the COVID-19 related rise in D-dimers significantly more than that associated with other lung infections? This could only be answered by conducting a study comparing D-dimer levels in COVID-19 related and nonCOVID-19 related lung infections, which to date has not been reported.

A rise in D-dimers and their increasing levels in the course of COVID-19 related illness has been proven to be associated with a considerable increase in morbidity and mortality [12-16].

\section{- LUPUS ANTICOAgULANT IN COVID - 19}

A prolonged aPTT was associated with COVID-19 in a small subgroup of patients [12]. In general, a prolongation of aPTT means one of the following, either a clotting factor deficiency or presence of circulating inhibitors of coagulation, and concerning the latter, the important one is the presence of lupus anticoagulant (LAC) [21].

In a study looking at the prolongation of aPTT and the presence of LAC in COVID-19 patients, of the 34 patients with prolonged aPTT, 31 patients (91\%) were positive for LAC [21].

Another study, evaluating the presence of LAC in COVID-19 patients, reported an incidence rate of $45 \%$ [22].

Helms et al. (2020) reported an incidence rate of $87.7 \%$ for a positive LAC in 50 of 57 tested patients [17].

From these studies, it can be postulated that the rise in aPTT in COVID-19 patients is related to the presence of LAC and that a prolonged aPTT, which would otherwise be worrying as a bleeding risk, may not be a problem in anticoagulating such patients, as they are already at a high risk of thromboses.

\section{anticoagulation in COVID - 19}

From the available evidence, it is clear that there are derangements in the coagulation parameters and increased incidence of thrombotic events in COVID-19 patients [12]. 
Table 1. Summary of important studies looking at haematological parameters in COVID - 19 patients. (aPTT - Activated Partial Thromboplastin time, FDP - Fibrin degradation products, PT - Prothrombin Time)

\begin{tabular}{|c|c|c|}
\hline Authors & Sample Size (n) & $\begin{array}{l}\text { Haematological } \\
\text { Abnormalities (\%) }\end{array}$ \\
\hline $\begin{array}{l}\text { Chen et al. } \\
\text { [29] }\end{array}$ & 99 & $\begin{array}{l}\text { Elevated D-dimer- } 36 \text { (36\%) } \\
\text { Thrombocytopenia - } 12(12 \%) \\
\text { Prolonged aPTT - } 6(6 \%) \\
\text { Prolonged PT - } 5(5 \%)\end{array}$ \\
\hline $\begin{array}{l}\text { Wang et } \\
\text { al. [30] }\end{array}$ & $\begin{array}{l}138 \\
(I C U-36 \\
\text { Non-ICU - 102) }\end{array}$ & $\begin{array}{l}\text { Prolonged PT }-80 \text { ( } 58 \%) \\
\text { Elevated D-dimer- } \\
\text { ( } 26 \% \text { of the patients from ICU) }\end{array}$ \\
\hline
\end{tabular}
Key Features

The first study to report both the clinical and laboratory features of COVID-19 related illness.

1 - The levels of D-dimer were significantly higher in ICU patients
than non-ICU patients. $(p<0.001)$
$2-$ The levels of D-dimer were significantly higher in non-survivors than survivors. $(p<0.05)$

3 - D-dimer levels showed an increasing trend in patients who succumbed to the illness.

\begin{tabular}{lll}
\hline Zhou et al. & 191 & Elevated D-dimer \\
{$[31]$} & (Survivors-137, & Survivors - 67 (57\%) \\
& Non-survivors & Non-survivors - 50 (92\%) \\
& $-54)$ & \\
\hline Huang et & 41 & - \\
al. [32] & $(I C U-13$, & \\
& Non-ICU - 28)
\end{tabular}

1 - A D-dimer level of $>1.0 \mu \mathrm{g} / \mathrm{mL}$ at admission was associated with higher odds of mortality. $O R=18.42(p=0.0033)$

2 - D-dimer levels were significantly higher in non-survivors than in survivors. (5.2 vs. 0.6, $p<0.0001$ )

1- Median D-dimer levels were significantly higher in ICU patients as compared to the non-ICU patients.

( 2.4 vs. $0.5, p=0.0042$ )

2 - Median prothrombin time was significantly higher in ICU patients as compared to the non-ICU patients.

(12.2sec vs. $10.7 \mathrm{sec}, \mathrm{p}=0.012$ )

Chen et al. 21

[33] (Severe cases -

11, Moderate

Compared to moderate cases, severe cases had significantly elevated levels of D-dimer. cases - 10)

( 2.6 vs. $0.3, p=0.029$ )

\begin{tabular}{ll}
\hline Guan et al. 1099 & Elevated D-dimer \\
[9] & $260 / 560(46.4 \%)$
\end{tabular}

Han et al. 94 patients

[34] 40 healthy controls
D-dimer levels were significantly elevated in a higher proportion of patients with severe illness than those with non-severe illness. (59.6\% vs. $43.2 \%, p=0.0021$ )

1- D-dimer levels were significantly higher in the patient group than the healthy control group.

(10.36 vs. $0.26, p<0.001$ )

2 - FDP levels were significantly higher in patients than in controls.

(33.83 vs. $1.55 \mathrm{mg} / \mathrm{L}, \mathrm{p}<0.001$ )

3- Higher D-dimer and FDP levels were found to be predictive of severe disease.

\begin{tabular}{ll}
\hline Li et al & 279 \\
{$[35]$} & (Ordinary- 136 \\
& Improved- 23 \\
& Poor- 120)
\end{tabular}

The D-dimer levels on admission were significantly higher in the improved and poor group of patients than ordinary patients. $(p<0.01)$

Ordinary - Mild disease, subsidedlmproved - First deteriorated, then improved gradually with treatment

Poor - Deteriorated or died

Tang et al. 183

[12] (Survivors - 162,

Non-survivors $-21)$

Abnormal coagulation tests (Elevated D-dimer, FDPs and decreased fibrinogen) were associated with a poorer prognosis, i.e. these parameters were significantly deranged in non-survivors than the survivors.

There are many clinical trials underway, testing the effectiveness of anticoagulation in COVID-19 patients (Clinical Trials.gov identifiers: NCT04345848, NCT04344756, NCT04359277).

A retrospective cohort study, including severely ill COVID-19 patients, looked at the twenty-eight daymortality in heparin users vs non-users. The results of this study showed a decreased twenty-eight day-mortality in heparin users in specific subgroups of patients with either sepsis-induced coagulopathy (SIC) score of $\geq 4$ or those having D-dimer levels $>6$ times of the upper limit of normal [23]. It was concluded that anticoagulation might be beneficial in individual patients with COVID-19, especially those with a SIC score of $\geq 4$ or those with markedly high D-dimer levels.

Table 2 summarises the societies and forums that have published interim guidance for managing coagulopathy in hospitalized COVID-19 patients. 


\section{AgentS SuPPRESSING ENDOTHELIAL INFLAMMATION -}

The efficacy of anticoagulants in preventing macrovascular thromboses (VTE) is well studied. However, their effectiveness in limiting microvascular thromboses is inconclusive [36, 37].

To prevent a microvascular thrombotic event, traditionally agents like corticosteroids, tocilizumab, complement inhibitors such as eculizumab, and Janus kinase inhibitors have been used in various autoimmune conditions as suppressants of endothelial inflammation [24].

Table 2. Summary of the interim guidelines published by some of the societies and forums. (aPTT - Activated Partial Thromboplastin Time, DOAC - Direct Oral Anticoagulant, ESC - European Society of Cardiology, ISTH - International Society on Thrombosis and Haemostasis, LMWH - Low Molecular Weight Heparin, VTE - Venous Thromboembolism)

Society/Forum

European Society of

Cardiology

(ESC) [25]
Tocilizumab (ClinicalTrials.gov identifiers: NCT043 20615, NCT04317092, NCT04363853) and corticosteroids (ClinicalTrials.gov identifier: NCT04273321) are currently being studied for treatment in COVID-19 associated illnesses. Use of this category of drugs in carefully selected COVID-19 patients may prove to be useful in preventing microvascular complications of the disease.

\section{RECOMMENDATIONS}

Derangements in coagulation parameters increased frequency of thrombotic events, and evidence of morRecommendations

1 - All admitted patients with COVID-19 related illnesses should get, at the least, prophylactic dose of enoxaparin (40mg daily).

2- Depending on the clinical features, a patient at a high risk of thromboembolism should receive therapeutic dose anticoagulation. It can be in the form of a heparin drip (per parenteral protocol) or enoxaparin ( $1 \mathrm{mg} / \mathrm{kg}$ twice a day) based on whether the patient is in the intensive care unit or not.

3- Patients at low risk of thromboembolism are further classified based on the D-dimer levels.

A) D-dimer $<0.5 \mu \mathrm{g} / \mathrm{mL}=$ Prophylactic dose anticoagulation (Enoxaparin 40mg/day)

B) D-dimer 0.5 to $3.0 \mu \mathrm{g} / \mathrm{mL}=$ Enoxaparin $40 \mathrm{mg}$ twice a day

C) D-dimer $>3.0 \mu \mathrm{g} / \mathrm{mL}=$ Enoxaparin $1 \mathrm{mg} / \mathrm{kg}$ twice a day

4- The patients at high risk of thromboembolism and having markedly elevated D-dimer $(>3.0 \mu \mathrm{g} / \mathrm{mL})$ should undergo a point-of-care ultrasound. Based on its results, a call should be on whether to continue therapeutic dose or switch to prophylactic dose anticoagulation.

International society 1- Patients having one/more of the following should be admitted to the hospital.

of thrombosis and $\quad$ A) Markedly raised D-dimer (>3-4 times of the normal)

haemostasis (ISTH) on B) Prolonged prothrombin time

the management of $\quad$ C) Platelet count of $<100 \times 10^{9} / \mathrm{L}$

coagulopathy [26] $\quad$ D) Fibrinogen concentration $<2.0 \mathrm{~g} / \mathrm{L}$

2-All admitted patients, in the absence of contraindications- should receive prophylactic dose anticoagulation (LMWH).

Scientific and stan- $\quad$ 1- Universal routine thromboprophylaxis should be given in all admitted patients of COVID-19 related illnesses. dardization committee (LMWH as preferred agent)

by ISTH-guidance on 2-Dose should be modified appropriately in patients with renal failure and obesity as required.

prevention and treat- $\quad 3$ - Intermediate dose anticoagulation can be a reasonable option in patients admitted to ICU with COVID-19 ment of VTE [27]

related illnesses.

4- Extended post-discharge thromboprophylaxis should be considered in patients that are a high risk of thromboembolism. The duration can be up to 30 days post-discharge.

Interim clinical guid-

1- All hospitalized patients, with COVID-19 related illnesses, should receive prophylactic anticoagulation.

ance from the antico-

2- Escalated dose anticoagulation should be considered in critically ill (ICU) patients.

agulation forum [28]

3- To monitor the anticoagulant activity of heparin, an anti-factor-Xa assay should be used in place of aPTT as the baseline aPTT may be abnormal in some COVID-19 patients.

4 - Post-discharge VTE prophylaxis may be considered on a case-to-case basis in COVID-19 patients that have one/more of the following-

A) Prolonged ICU stay

B) Paralyzed for a long time

C) Risk factor for VTE at the time of discharge (Decreased mobility, severe weakness)

5 - All pregnant patients of COVID-19 should receive prophylactic dose anticoagulation for the prevention of VTE.

6 - Patients on vitamin $\mathrm{K}$ antagonists (warfarin) should be transitioned to directly acting oral anticoagulants (DOACs), except for indications like mechanical heart valves, antiphospholipid antibody syndrome. 
tality benefit in critically ill make a strong case for exploring the efficacy of anticoagulants in COVID-19 patients. While randomized controlled trials are ideal, the rapid spread and relatively high incidence of VTE events in COVID-19 patients temporarily preclude their need for formulating working clinical guidelines. Based on the interpretation of the available literature, it is recommended that:

1. Prophylactic dose anticoagulation to be given to all patients admitted to a hospital due to $\mathrm{CO}$ VID-19 related illness.

2. Therapeutic anticoagulation to be prescribed for patients having markedly elevated D-dimer levels and patients that are paralyzed or bedridden.

3. An intermediate escalated-prophylactic anticoagulation regime be prescribed for all patients admitted to an ICU and those having clinical features suggestive of ARDS.

4. Serial monitoring of D-dimers to be prescribed carried out to stratify patients and provide an appropriate line of management.

\section{CONCLUSION}

COVID-19 associated coagulopathy is associated with significant morbidity and mortality. Evidence of the development of coagulopathy is gained from basic blood investigations monitoring D-dimer, FDPs, and fibrinogen levels.

Many societies and forums have recommend the use of prophylactic dose anticoagulation in all hospitalized patients with COVID-19, and especially in critically ill patients. Reports in the literature support this approach.

\section{CONFLICT OF INTEREST}

None to declare.

\section{- REFERENCES}

1. WuF, Zhao S, Yu B, et al. A new coronavirus associated with human respiratory disease in China. Nature. 2020;579(7798):265-269. doi:10.1038/s41586-020-2008-3

2. Bikdeli B, Madhavan M V., Jimenez D, et al. COVID-19 and Thrombotic or Thromboembolic Disease: Implications for Prevention, Antithrombotic Therapy, and Follow-up. J Am Coll Cardiol. 2020;75(23). doi:10.1016/j.jacc.2020.04.031

3. Connors JM, Levy JH. COVID-19 and its implications for thrombosis and anticoagulation. Blood. 2020;135(23):20332040. doi:10.1182/blood.2020006000

4. Grasselli G, Pesenti A, Cecconi M. Critical Care Utilization for the COVID-19 Outbreak in Lombardy, Italy: Early Experience and Forecast during an Emergency Response. JAMA- J Am Med Assoc. Published online, 2020. doi:10.1001/jama.2020.4031

5. Yang $X, Y u Y, X u J$, et al. Clinical course and outcomes of critically ill patients with SARS-CoV-2 pneumonia in Wuhan, China: a singlecentred, retrospective, observational study. Lancet Respir Med. 2020;8(5):475-481. doi:10.1016/S2213-2600(20)30079-5

6. Bhatraju PK, Ghassemieh BJ, Nichols M, et al. Covid-19 in Critically III Patients in the Seattle Region - Case Series. N Engl J Med. Published online 2020:2012-2022. doi:10.1056/ nejmoa2004500

7. Engelmann B, Massberg S. Thrombosis as an intravascular effector of innate immunity. Nat Rev Immunol. 2013;13(1):3445. doi:10.1038/nri3345

8. Delabranche X, Helms J, Meziani F. Immunohaemostasis: a new view on haemostasis during sepsis. Ann Intensive Care. 2017;7(1):1-14. doi:10.1186/s13613-017-0339-5

9. Guan $\mathrm{W}, \mathrm{Ni} Z, \mathrm{Hu}$ Y, et al. Clinical characteristics of coronavirus disease 2019 in China. N Engl J Med. 2020;382(18):1708-1720. doi:10.1056/NEJMoa2002032

10. Klok FA, Kruip MJHA, van der Meer NJM, et al. incidence of thrombotic complications in critically ill ICU patients with COVID-19. Thromb Res. 2020;191(April):145-147. doi:10.1016/j.thromres.2020.04.013

11. Fox SE, Akmatbekov A, Harbert JL, Li G, Brown JQ. Pulmonary and Cardiac Pathology in Covid-19: The First Autopsy Series from New Orleans 1 ) Department of Pathology, LSU Health Sciences Center, New Orleans 2 ) Pathology and Laboratory Medicine Service, Southeast Louisiana Veterans Healthcare System 3. medRxiv. Published online, 2020.

12. Tang N, Li D, Wang X, Sun Z. Abnormal coagulation parameters are associated with poor prognosis in patients with novel coronavirus pneumonia. J Thromb Haemost. 2020;18(4):844847. doi:10.1111/jth.14768

13. Klok FA, Kruip MJHA, van der Meer NJM, et al. Confirmation of the high cumulative incidence of thrombotic complications in critically ill ICU patients with COVID-19: An updated analysis. Thromb Res. 2020;191(April):148-150. doi:10.1016/j. thromres.2020.04.041

14. Al-Samkari H, Karp Leaf RS, Dzik WH, et al. COVID and Coagulation: Bleeding and Thrombotic Manifestations of SARSCoV2 Infection.; 2020. doi:10.1182/blood.2020006520

15. Cui S, Chen S, Li X, Liu S, Wang F. Prevalence of venous thromboembolism in patients with severe novel coronavirus pneumonia. J Thromb Haemost. 2020;(April):1421-1424. doi:10.1111/jth.14830

16. Hippensteel JA, Burnham EL, Jolley SE. Prevalence of Venous Thromboembolism in Critically III Patients with COVID-19. Br J Haematol. Published online 2020:0-3. doi:10.1111/bjh.16908

17. Helms J, Tacquard C, Severac F, et al. High risk of thrombosis 
Available online at: www.jccm.ro

in patients with severe SARS-CoV-2 infection: a multicenter prospective cohort study. Intensive Care Med. Published online, 2020. doi:10.1007/s00134-020-06062-x

18. Llitjos JF, Leclerc $M$, Chochois $C$, et al. High incidence of venous thromboembolic events in anticoagulated severe COVID-19 patients. J Thromb Haemost. 2020;(April):1-4. doi:10.1111/ jth.14869

19. Nahum J, Morichau-Beauchant T, Daviaud F, et al. Venous ThrombosisAmong Critically III Patients With Coronavirus Disease 2019 (COVID-19). JAMA Netw Open. 2020;3(5):e2010478. doi:10.1001/jamanetworkopen.2020.10478

20. Lippi G, Bonfanti L, Saccenti C, Cervellin G. Causes of elevated D-dimer in patients admitted to a large urban emergency department. Eur J Intern Med. 2014;25(1):45-48. doi:10.1016/j. ejim.2013.07.012

21. Bowles L, Platton S, Yartey N, et al. Lupus Anticoagulant and Abnormal Coagulation Tests in Patients with Covid-19. N Engl J Med. Published online 2020:1-2. doi:10.1056/nejmc2013656

22. Harzallah I, Debliquis A, Drénou B. Lupus anticoagulant is frequent in patients with Covid-19. J Thromb Haemost. 2020;(April):14867. doi:10.1111/jth.14867

23. Tang N, Bai H, Chen X, Gong J, Li D, Sun Z. Anticoagulant treatment is associated with decreased mortality in severe coronavirus disease 2019 patients with coagulopathy. J Thromb Haemost. 2020;(March):1094-1099. doi:10.1111/jth.14817

24. Wiseman AC. Immunosuppressive medications. Clin J Am Soc Nephrol. 2016;11(2):332-343. doi:10.2215/CJN.08570814

25. Journal EH, Pharmacotherapy C. Figure 1. Published online 2020:1-2. doi:10.1111/jth.14817.6.

26. Thachil J, Tang N, Gando S, et al. ISTH interim guidance on recognition and management of coagulopathy in COVID-19. J Thromb Haemost. 2020;(March):1023-1026. doi:10.1111/ jth.14810

27. Spyropoulos AC, Levy JH, Ageno W, et al. Scientific and Standardization Committee Communication: Clinical Guidance on the Diagnosis, Prevention and Treatment of Venous Thromboembolism in Hospitalized Patients with COVID-19. J Thromb Haemost. Published online 2020:0-2. doi:10.1111/ jth.14929
The Journal of Critical Care Medicine 2020;6(4) • 223

28. Barnes GD, Burnett A, Allen A, et al. Thromboembolism and anticoagulant therapy during the COVID-19 pandemic: interim clinical guidance from the anticoagulation forum. J Thromb Thrombolysis. 2020;(0123456789):1-10. doi:10.1007/s11239020-02138-z

29. Chen N, Zhou M, Dong X, et al. Epidemiological and clinical characteristics of 99 cases of 2019 novel coronavirus pneumonia in Wuhan, China: a descriptive study. Lancet. 2020;395(10223):507-513. doi:10.1016/S01406736(20)30211-7

30. Wang D, Hu B, Hu C, et al. Clinical Characteristics of 138 Hospitalized Patients with 2019 Novel Coronavirus-Infected Pneumonia in Wuhan, China. JAMA - J Am Med Assoc. 2020;323(11):1061-1069. doi:10.1001/jama.2020.1585

31. Zhou F, Yu T, Du R, et al. Clinical course and risk factors for mortality of adult inpatients with COVID-19 in Wuhan, China: a retrospective cohort study. Lancet. 2020;395(10229):10541062. doi:10.1016/S0140-6736(20)30566-3

32. Huang C, Wang $Y$, Li $X$, et al. Clinical features of patients infected with 2019 novel coronavirus in Wuhan, China. Lancet. 2020;395(10223):497-506. doi:10.1016/S01406736(20)30183-5

33. Chen G, Wu D, Guo W, et al. Clinical and immunological features of severe and moderate coronavirus disease 2019. J Clin Invest. 2020;130(5):2620-2629. doi:10.1172/JCl137244

34. Han $H$, Yang $L$, Liu $R$, et al. Prominent changes in blood coagulation of patients with SARS-CoV-2 infection [published online ahead of print, 2020 Mar 16]. Clin Chem Lab Med. Published online, 2020. doi:10.1515/cclm-2020-0188

35. Li Y, Zhao K, Wei $H$, et al. Dynamic relationship between Ddimer and COVID-19 severity. Br J Haematol. Published online 2020:bjh.16811. doi:10.1111/bjh.16811

36. Ashjian P, Chen CM, Pusic A, Disa JJ, Cordeiro PG, Mehrara BJ. The effect of postoperative anticoagulation on microvascular thrombosis. Ann Plast Surg. 2007;59(1):36-39. doi:10.1097/01. sap.0000264837.15110.2f

37. Sorg H, Hoffmann JO, Hoffmann JN, Vollmar B. Analysis of the influence of antithrombin on microvascular thrombosis: antiinflammation is crucial for anticoagulation. Intensive Care Med Exp. 2015;3(1):22. doi:10.1186/s40635-015-0058-x 